\title{
Disconnecting Surgical Treatment of Hypothalamic Hamartoma in Children and Adults With Refractory Epilepsy and Proposal of a New Classification
}

\author{
Olivier DELALANDE and Martine FOHLEN
}

Unite de neurochirurgie pédiatrique, Fondation Ophtalmologique A. de Rothschild, Paris, France

\begin{abstract}
A series of 17 patients aged from 9 months to 32 years with refractory epilepsy due to hypothalamic hamartoma were treated by total removal (one case) and disconnection (16 cases) between 1997 and 2002. The mean age at seizure onset was 16 months. Sixteen patients had gelastic seizures, 14 had partial seizures and three had generalized tonic-clonic seizures. The mean seizure frequency was 21 per day. Four patients had borderline intelligence quotient and the others were mentally retarded. Five patients presented with precocious puberty, one with acromegaly, and four suffered from obesity. Brain magnetic resonance imaging, performed at least twice in each patient, showed the hamartoma as a stable homogeneous interpeduncular mass implanted either on the mammilary tubercle or on the wall of the third ventricle with variable extension to the bottom. Ictal single photon emission computed tomography, performed in four patients, showed hyperperfusion within the hamartoma in two patients. Twenty-five operations were performed in the 17 patients. The first patient underwent total removal of the hamartoma, whereas the following 16 patients underwent disconnection through open surgery (14 procedures) and/or endoscopy ( 9 procedures). Eight patients became seizure-free, one patient had only brief gelastic seizures, and eight patients were dramatically improved with a mean follow up of 18.6 months (8 days to 43 months). Surgery was safe in all but two patients: the first patient had transient hemiplegia and the third cranial nerve paresis, and the other developed hemiplegia due to ischemia of the middle cerebral artery territory. The quality of life, and behavior and school performance were greatly improved in most patients. Our series illustrates the feasibility and relative safety of disconnection surgery for hypothalamic hamartomas with seizure relief in $53 \%$ of patients and dramatic improvement in the others. Surgical observations led us to propose a new anatomical classification according to the anatomical relationship between the hamartoma and the adjacent hypothalamus and third ventricle. Endoscopic disconnection seems to be a very safe way to treat hamartomas in intraventricular locations.
\end{abstract}

Key words: hypothalamic hamartoma, gelastic epilepsy, disconnecting surgery, children

\section{Introduction}

Hypothalamic hamartomas are rare congenital lesions consisting of mixed neurons and glial cells ${ }^{9)}$ that usually take the form of nodules located on the wall or the floor of the third ventricle or attached to

Hypothalamic hamartoma associated with intractable epilepsy is a major problem when surgical management is discussed. Professor Olivier Delalande is Chief Neurosurgeon of the Department of Pediatric Neurosurgery, Fondation Ophtalmologique Adolf de Rothschild, in Paris, France. In recent international symposia, he has reported a series of hypothalamic hamartoma in children and adults with refractory epilepsy, managed with various surgical techniques according to his new classification of hypothalamic hamartomas. With the permission of Professor Akira Yamaura, Chief Editor of the Neurologia medico-chirurgica, this stimulating paper has appeared in this issue. The strategies of his surgery using surgical excision, surgical disconnection or endoscopic disconnection are challenging but safe. His surgical outcomes are that 8 patients are seizure free, one patient has only brief gelastic seizures and 8 patients are dramatically improved. I hope that the authors will have the opportunity to again examine the outcomes in their series several years from now.

Tatsuya TANAKA, M.D., D.M.Sc., President of the 30th Annual Meeting of the Japan Society for Pediatric Neurosurgery. 
the tuber cinereum or mammillary bodies. Hypothalamic hamartomas are often associated with an epileptic syndrome which usually begins in early childhood with gelastic seizures and later continues as generalized epileptic encephalopathy characterized by other types of seizures (partial and generalized) and associated with cognitive decline and behavioral disorders. ${ }^{4,17)}$ The epilepsy is frequently refractory to antiepileptic drugs. Depth electrode recordings have demonstrated that gelastic seizures arise within the hamartoma, ${ }^{1,3,5,7,11,13)}$ so resection of the hamartoma seems to be the most appropriate treatment.

Recently, various therapeutic approaches for epilepsy have been attempted with encouraging results, including gamma knife surgery, ${ }^{2,18,22)}$ radiofrequency thermocoagulation, ${ }^{7)}$ and chronic electrical stimulation of the hamartoma and microsurgery using different routes. ${ }^{14,15,20,23)}$ Most previous studies have included single cases or series, some of precocious puberty only, others with epilepsy only or both. ${ }^{10,12)}$

We recently reported disconnection of hamartoma of the fourth ventricle which resulted in total relief of seizures in two patients. ${ }^{6)}$ In the present study, we performed disconnection of the hamartoma using either open cranial surgery or endoscopic surgery guided with a frameless stereotactic robot in a series of 17 children and adolescents referred for intractable epilepsy due to hypothalamic hamartoma, and propose a classification for hamartoma to aid in the selection of the surgical treatment.

\section{Materials and Methods}

\section{Patients}

Seventeen patients, 12 males and five females aged at surgery from 9 months to 32 years (mean 13.8 years), were treated for refractory epilepsy associated with hypothalamic hamartoma between January 1997 and January 2002 in our department (Table 1). Prior to surgery, the patients underwent neurological examination and neuropsychological assessment, routine electroencephalography (EEG) and video-EEG monitoring, magnetic resonance (MR) imaging, and endocrinological investigations.

The onset of the epilepsy ranged from the neonatal period to 24 months (mean 16 months) and the age at diagnosis of hypothalamic hamartoma ranged from 1 month to 10 years (mean 3 years). The seizure types were classified as follows: infantile spasms (1/17 patients), gelastic seizures (16/17), dacrystic seizures (2/17), partial seizures (14/17), tonic seizures (8/17), atonic seizures (2/17), and generalized tonic clonic seizures (3/17). Eight of 17 patients experienced three seizure types, seven patients had two seizure types, and two patients had four seizure types. The seizure frequency ranged from six seizures per hour to four seizures weekly (mean 21 seizures daily).

Neurological examination showed no motor deficit in any patient. One patient had congenital deafness and was classified as Pallister-Hall syndrome (Case 4). Neuropsychological examination performed at the time of the operation showed border line intelligence quotient (IQ) in four patients, mild mental deficiency (IQ 40-80) in six, and severe mental deficiency (IQ $<40$ ) in five. The two infants had developmental quotients of 64 and 60. Five children among the most mentally impaired patients (Cases 1, 4, 8, and 10) had behavioral problems that encompassed hyperkinesia, aggressiveness, and psychotic features. One patient had frontal syndrome (Case 16) and another had anxiety disorders (Case 7). The other patients presented with no major behavioral disorders.

Endocrinological disorders were observed in nine patients; four had polyphagia associated with obesity, five had precocious puberty, and one had gigantism associated with acromegaly and hypothyroidism. None of our patients had any visual impairment.

MR imaging was performed at least twice in each patient. All hamartomas were stable lesions appearing as iso-intense on $\mathrm{T}_{1}$-weighted images without enhancement by contrast medium and iso- or hyperintense on $\mathrm{T}_{2}$-weighted images. All hamartomas were sessile with variable attachment to the hypothalamus and great variation in size. Ictal single photon emission computed tomography was performed in four patients, showing hyperperfusion within the lesion in two patients and no abnormalities in the other two patients.

\section{Classification of hamartomas and surgical procedures}

Twenty-five operations were performed in the 17 patients, as eight patients required reoperations (Table 2).

Case 1 underwent complete resection of the lesion through a pterional route. Postoperatively, he became seizure-free (Engel class I) but developed partially regressive right hemiplegia and left third cranial nerve paresis. These complications were unforeseen considering the operative conditions. Clinical signs and postoperative MR imaging suggested a vascular complication. Therefore, to avoid further complications, we decided to perform disconnection associated with variable resection in the subsequent patients.

Various surgical procedures have been evolved for 
Table 1 Clinical summary of cases

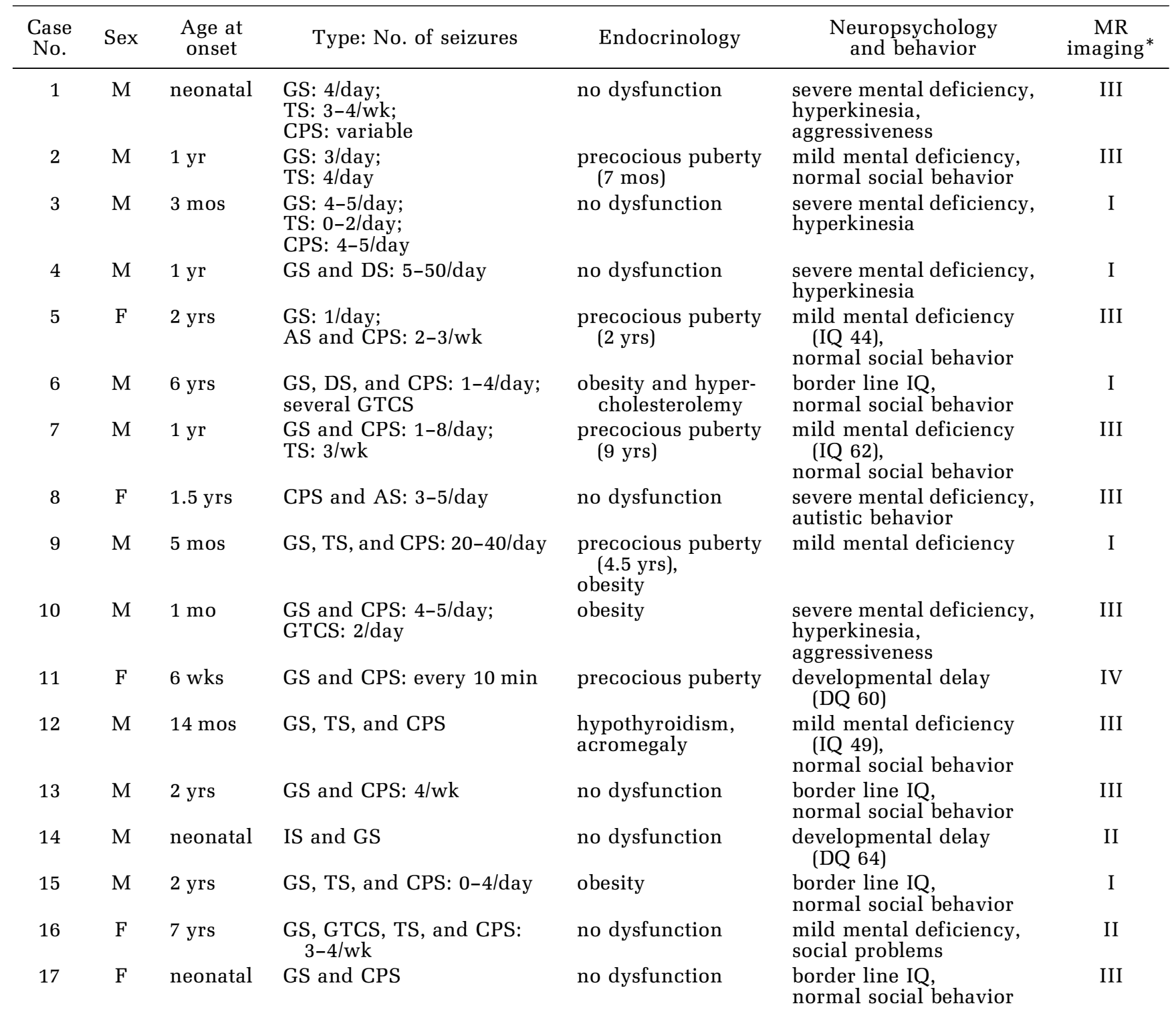

${ }^{*}$ Type of personal classification. AS: absence seizure, CPS: complex partial seizure, DQ: developmental quotient, DS: dacrystic seizure, GS: gelastic seizure, GTCS: generalized tonic-clonic seizure, IQ: intelligence quotient, IS: infantile spasm, TS: tonic seizure.

the anatomical conditions of hamartomas. We have developed a classification for hamartomas based on the plane of insertion on the hypothalamus to help choose the best surgical route (Fig. 1).

Hamartoma Type I has a horizontal implantation plane and may be lateralized on one side; the microsurgical route should be pterional leading to complete disconnection (Cases 3, 4, 6, 9, and 15).

Hamartoma Type II has a vertical insertion plane and intraventricular location; the surgical procedure consists of disconnection of the intraventricular component of the hamartoma using an endoscop- ic route with a frameless stereotactic robot and the endoscopic resection can be repeated if required (Cases 14 and 16).

Hamartoma Type III is a combination of Types I and II; we recommend a two-step procedure: the first step consists of disconnection of the intraventricular part of the hamartoma using an endoscopic route and the second step consists of disconnection through a pterional route (Cases 1, 2, 5, 7, 8, 10, 12, 13, and 17).

Hamartoma Type IV includes all giant hamartomas for which no specific surgical procedures can 
Table 2 Surgical procedures

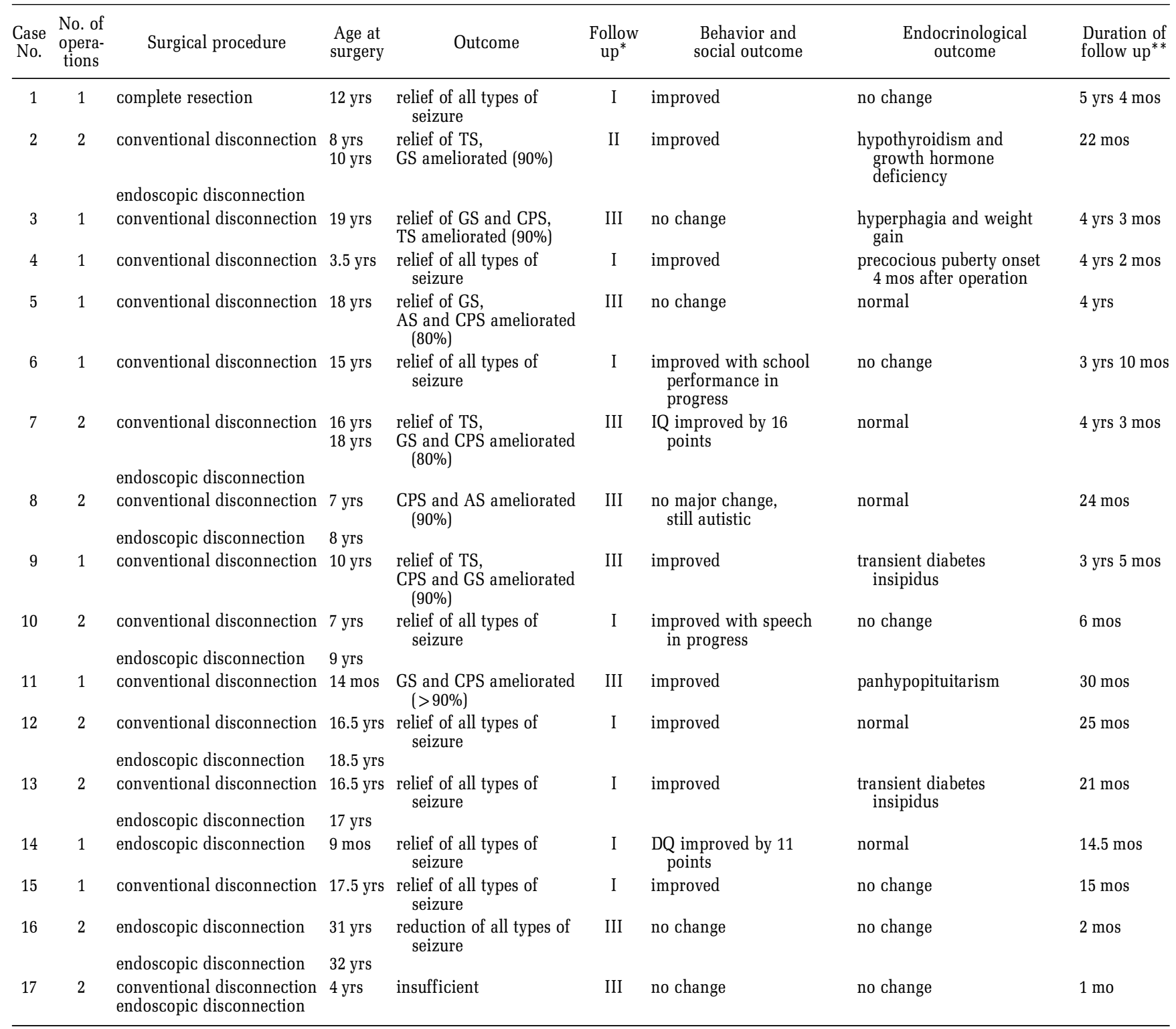

${ }^{*}$ Engel class. ${ }^{* *}$ Since the last operation. AS: absence seizure, CPS: complex partial seizure, DQ: developmental quotient, GS: gelastic seizure, IQ: intellectual quotient, TS: tonic seizure.

be recommended (Case 11).

According to our classification, 14 patients underwent disconnection through a pterional route and nine patients $(7$ reinterventions and 2 primary interventions) underwent endoscopic surgery with the aid of a frameless stereotactic robot.

Postoperative complications consisted of meningitis (Case 3), hemiplegia due to ischemia of the middle cerebral artery territory (Case 5), ischemic lesion of the internal part of the frontal lobe (Case 13), and transient diabetes insipidus (Cases 9 and 13). None of the patients who underwent endoscopic discon- nection suffered any postoperative complications and all were discharged only 3 days after the operation.

\section{Results}

\section{Effect on seizures}

The completeness of disconnection of the hamartoma is difficult to assess during the operation because of the lack of clear-cut borders between the lesion and the hypothalamus, so the effect on the epilepsy remains the only indicator of adequate dis- 

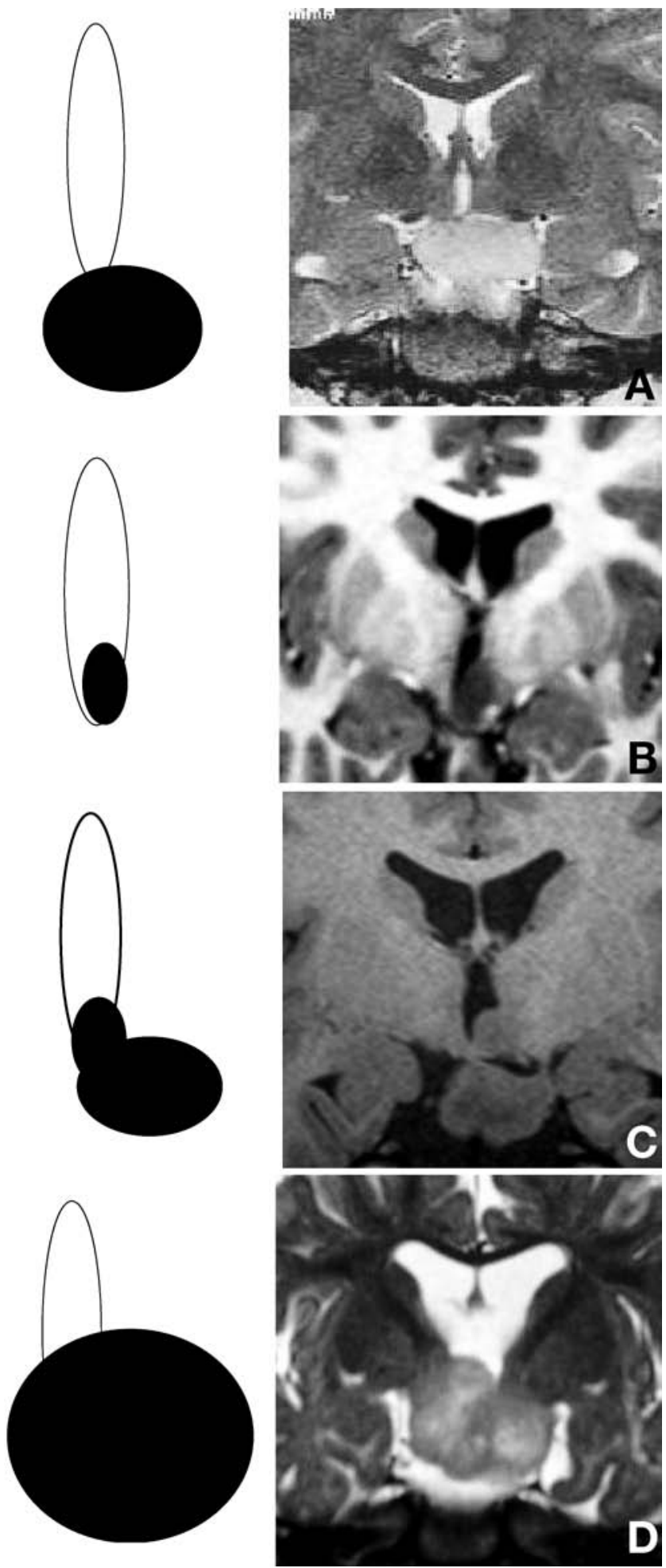

Fig. 1 Anatomical classification. A: Type I, B: Type II, C: Type III, D: Type IV. connection.

The first patient (Case 1) became seizure-free (follow up 5 years) and demonstrated dramatic improvement of behavior and language performance. Antiepileptic drugs were withdrawn 2 years after the operation.

The 14 patients who underwent open surgery had the following outcomes: three patients became seizure-free (Engel class I), and 11 showed a dramatic reduction of frequency $>80 \%$ of all types of seizures (Engel class III) with total disappearance of tonic and generalized tonic-clonic seizures in five patients. Seven of these 11 patients underwent an additional endoscopic disconnection with a follow up of 3 months to 4 years (mean 21 months). Three patients became seizure-free (Cases 10, 12, and 13), one had only gelastic seizures (Case 2), and three showed reduced seizure frequency (Cases 7, 8, and 17).

Two patients underwent only endoscopic disconnection; one became seizure-free (Case 14) and the other was Engel class III (Case 16) after the first operation and was recently reoperated with no marked improvement (follow up 6 months).

The outcomes for all surgical approaches with a mean follow up of 18.6 months ( 8 days to 43 months) were seizure-free in eight patients, only brief gelastic seizures (Engel class II) in one patient, and dramatically improved (Engel class III) in eight patients.

\section{Other results}

Behavior, as evaluated by the families of the patient and their physicians, and school performance were improved in Engel class I to III patients. No patient experienced any cognitive aggravation. One patient obtained a driving license, two patients are learning manual skills, and one patient (class II) has an improved IQ of 16 points 15 months following surgery.

Two patients had definitive postoperative neurological deficits. Endocrinological follow-up examination showed hyperphagia with weight gain in one patient, hypothyroidism in one, and no change in the others.

\section{Histopathology}

Histological examination of the tumor samples found neuronal tissue made of scattered neurons and glial cells. The cellularity was sometimes increased or normal. The glial cells were mainly microglia. The neuronal cells were sometimes ganglionic neurons and in some cases the neurons had a degenerative aspect characterized by loss of basophilia of the cytoplasm. 


\section{Discussion}

\section{Surgical approaches and techniques}

Various operative routes for the surgical treatment of hypothalamic hamartomas have achieved various degrees of reduction of epilepsy. ${ }^{8,14,15,20,23)}$ In all cases the goal of the treatment was the removal of the entire lesion since it is commonly agreed that the hamartoma is the trigger of the epilepsy $3,5,7,8,11,13,21)$ and so the resection of hypothalamic hamartoma must be as complete as possible to achieve good seizure remission. ${ }^{16)}$

The present study suggests that complete removal of the hamartoma can be replaced by disconnection, using either open surgery or endoscopy, since the hamartoma is a stable lesion and entire anatomical removal is not necessary to treat the epilepsy. Such a surgical method was first applied to epilepsy in the form of hemispherotomy ${ }^{24)}$ instead of hemispherectomy with similar results upon the outcome of seizures, with corresponding reduction in the operative risks and postoperative complications. More recently, we performed disconnection in two children with hamartoma of the fourth ventricle associated with facial hemispasms, with excellent outcome regarding the seizures. ${ }^{6)}$ Moreover, the endoscopic approach allows disconnection of the intraventricular part of the hamartoma and is very well tolerated by the patients; in case of failure or insufficient results, the procedure can be performed again. The problem is to define accurately the borders of the disconnection since there are no clear-cut limits between the hamartoma and the neighboring normal brain tissue.

Recently, a series of five children were treated with transcallosal resection. ${ }^{20}$ Three became seizure-free and two were improved. The only postoperative complications were endocrinological such as hyperphagia and transient diabetes insipidus. Our endoscopic route is very similar to this transcallosal approach but it seems to us that the transcallosal approach may be inadequate for cases in which the implantation of the hamartoma spreads horizontally below the hypothalamus (Delalande's Type I), for which we recommend open surgery with a pterional route.

Another challenge is to attempt to classify the hamartomas in order to define the best surgical procedure. In 1994, Valdueza et al. ${ }^{23)}$ proposed a classification for hamartomas into four types (Ia, Ib, IIa, and IIb) based on the anatomical and clinical data from 42 patients (personal cases and literature selection). Types Ia and Ib were defined as small pedunculated hamartomas attached to the tuber cinereum. Clinically the patients are either asympto- matic or have precocious puberty. Surgical removal is indicated only in the rare event of visual disturbance or to avoid long-term luteinizing hormonereleasing hormone analogue medication. Type II tumors consist of hamartomas larger than $1.5 \mathrm{~cm}$ with a sessile attachment to the floor of the third ventricle and the mammillary bodies without (IIa) or with (IIb) distortion of the third ventricle. Clinically the patients suffer from epilepsy associated with memory disturbances (IIa) and mental and behavior abnormalities (IIb). The surgical approach to type II hamartomas is recommended because of the failure to control the seizures with medication, but the operative route was not discussed.

Our 17 cases are consistent with Valdueza's type II as all had a sessile attachment to the hypothalamus with or without distortion of the wall of the third ventricle associated with epilepsy. We have proposed another classification of these cases into four types based on the anatomy only, as we could detect no differences in the severity of epilepsy or behavioral problems correlated with the size or implantation of the hypothalamic hamartomas. We suggest that the major feature in the planning of the optimum surgical management is the plane of attachment (horizontal, vertical, or both) of the lesion to the hypothalamus.

\section{Surgery or radiosurgery}

Radiosurgery appears to be an alternative treatment for hypothalamic hamartoma with epilepsy. In 2000, Regis et al. ${ }^{18)}$ reported a series of eight patients with sessile hypothalamic hamartoma and drugresistant epilepsy who were treated with gamma knife surgery (GKS). Four of these patients were cured or greatly improved and the remaining four patients exhibited marked improvement after a median latency in seizure cessation of 9 months. No side effects were reported within a median follow up of 28 months. The main argument advocated for radiosurgical treatment rather than microsurgery is the surgical risk of the resection of the hamartoma.

In our series, the first patient who underwent total resection of a large pediculate hamartoma experienced postoperative complications such as oculomotor nerve paresis and hemiplegia. Therefore, in the following consecutive patients, we decided to perform disconnection of the lesion rather than resection. With this approach, complications are infrequent, the effects on epilepsy quickly appear, and endoscopic disconnection can be repeated in the case of failure. In addition, patients do not suffer the long-term complications reported in patients undergoing GKS for mesial temporal lobe epilepsy. ${ }^{19)}$ Moreover, large implanted as well as 
small intraventricular lesions can be cured by disconnection, whereas Regis et al. ${ }^{18)}$ suggested that only small hypothalamic hamartomas located inside the third ventricle or in the floor are good candidates for GKS.

\section{Other treatment approaches}

In 1999, Fukuda et al. ${ }^{7)}$ reported a single patient with hypothalamic hamartoma treated by stereotactic radiofrequency thermocoagulation in the course of an exploration with a depth electrode implanted within the lesion, associated with subdural grids. Two gelastic seizures were recorded, originating from the hamartoma. The stimulation electrode was replaced by acoagulation electrode to treat the patient. Gelastic seizures ceased postoperatively whereas tonic seizures disappeared 4 months later. This patient is now seizure-free with a follow up of 14 months. To the best of our knowledge, this is the only case treated in that way and this technique seems to be suitable for Valdueza's Type II hamartomas.

\section{Conclusion}

Our series illustrates the feasibility and acceptable morbidity of disconnective surgery for treating hypothalamic hamartomas. This surgery is likely to become safer as technical procedures evolve. The safest method is stereotactic endoscopy indicated for cases with endo- or periventricular hypothalamic insertion. Since the prognosis of epilepsy linked to hypothalamic hamartomas is currently poor, surgical treatment has to be performed early, as soon as the epilepsy is identified as drug resistant, to prevent behavioral disorders and delayed development in children. Parents and patients must be aware that it is often necessary to operate twice or even more in cases of failure to control seizures and that disconnection performed under open surgery can be followed by endoscopic disconnection in cases of persistent seizures.

\section{References}

1) Arita K, Ikawa F, Kurisu K, Sumida M, Harada K, Uozumi T, Monden S, Yoshida J, Nishi Y: The relationship between magnetic resonance imaging findings and clinical manifestations of hypothalamic hamartoma. J Neurosurg 91: 212-220, 1999

2) Arita K, Kurisu K, Iida K, Hanaya R, Akimitsu T, Hibino S, Pant B, Hamasaki M, Shinagawa S: Subsidence of seizure induced by stereotactic radiation in a patient with hypothalamic hamartoma: case report. J Neurosurg 89: 645-648, 1998

3) Arroyo S, Santamaria J, Sanmarti F, Lomena F, Catafau A, Casamitjana R, Setain J, Tolosa E: Ictal laughter associated with paroxysmal hypothalamopituitary dysfunction. Epilepsia 38: 114-117, 1997

4) Berkovic SF, Andermann F, Melanson D, Ethier RE, Feindel W, Gloor P: Hypothalamic hamartoma and ictal laughter: evolution of a characteristic epileptic syndrome and diagnostic value of magnetic resonance imaging. Ann Neurol 23: 429-439, 1988

5) Berkovic SF, Kuzniecky RI, Andermann F: Human epileptogenesis and hypothalamic hamartomas: new lessons from an experiment of nature. Epilepsia 38: $1-3,1997$

6) Delalande O, Rodriguez D, Chiron C, Fohlen M: Successful surgical relief of seizures associated with hamartoma of the floor of the fourth ventricle in children: report of 2 cases. Neurosurgery 49: 726-731, 2001

7) Fukuda M, Kameyama S, Wachi M, Tanaka R: Stereotaxy for hypothalamic hamartoma with intractable gelastic seizures: Technical case report. Neurosurgery 44: 1347-1350, 1999

8) Gascino GD, Andermann F, Berkovic SF, Kuzniecky RI, Sharbrough FW, Keene DL, Bladin PF, Kelly PJ, Olivier A, Feindel W: Gelastic seizures and hypothalamic hamartomas: Evaluation of patients undergoing chronic intracranial EEG monitoring and outcome of surgical treatment. Neurology 43: 747-750, 1993

9) Hamilton RL: Case of the month, July 1996: precocious puberty. Brain Pathol 7: 711-712, 1997

10) Kammer KS, Perlman K, Humphreys RP, Howard NJ: Clinical and surgical aspects of hypothalamic hamartoma associated with precocious puberty in a 15month-old boy. Childs Brain 7: 150-157, 1980

11) Kuzniecky R, Guthrie B, Mountz J, Bebin M, Faught E, Gilliam F, Liu HG: Intrinsic epileptogenesis of hypothalamic hamartomas in gelastic epilepsy. Ann Neurol 42: 60-67, 1997

12) Mottolese C, Stan H, Bret P, Berlier P, Laprea C: Hypothalamic hamartomas: the role of surgery in a series of eight patients. Childs Nerv Syst 17: 229-236, 2001

13) Munari C, Kahane P, Francione S, Hoffmann D, Tassi L, Cusmai R, Vigevano F, Pasquier B, Betti OO: Role of hypothalamic hamartoma in the genesis of gelastic fits (a video-stereo-EEG study). Electroencephalogr Clin Neurophysiol 95: 154-160, 1995

14) Nishio S, Fujiwara S, Aiko Y, Takeshita I, Fukui M: Hypothalamic hamartoma; Report of two cases. J Neurosurg 70: 640-654, 1989

15) Nishio S, Morioka T, Fukui M, Goto Y: Surgical treatment of intractable seizures due to hypothalamic hamartoma. Epilepsia 35: 514-519, 1994

16) Palmini A, Chandler C, Andermann F, Costa Da Costa J, Paglioli-Neto E, Polkey C, Rosenblatt B, Montes J, Martinez JV, Farmer JP, Sinclair B, Aronyk K, Paglioli E, Coutinho L, Raupp S, Portuguez M: Resection of the lesion in patients with hypothalamic hamartomas and catastrophic epilepsy. Neurology 58: 1138-1147, 2002 
17) Ponsot G, Diebler C, Plouin P, Nardou M, Dulac O, Chaussain JL, Arthuis M: Hamartomes hypothalamiques et crises de rire: a propos de 7 observations. Arch Fr Pediatr 40: 757-761, 1983 (Fre, with Eng abstract)

18) Regis J, Bartolomei F, de Toffol B, Genton P, Kobayashi T, Mori Y, Takakura K, Hori T, Inoue H, Schrottner O, Pendl G, Wolf A, Arita K, Chauvel P: Gamma knife surgery for epilepsy related to hypothalamic hamartomas. Neurosurgery 47: 1343-1352, 2000

19) Regis J, Bartolomei F, Rey M, Genton P, Dravet C, Semah F, Gastaut JL, Chauvel P, Peragut JC: Gamma knife surgery for mesial temporal lobe epilepsy. Epilepsia 40: 1551-1556, 1999

20) Rosenfeld JV, Harvey AS, Wrennall J, Zacharin M, Berkovic SF: Transcallosal resection of hypothalamic hamartomas, with control of seizures, in children with gelastic epilepsy. Neurosurgery 48: 108-118, 2001

21) Tasch E, Cendes F, Li LM, Dubeau F, Montes J, Rosenblatt B, Andermann F, Arnold D: Hypothalamic hamartomas and gelastic epilepsy: a spectroscopic study. Neurology 51: 1046-1050, 1998

22) Unger F, Schrottner, Haselsberger K, Korner E, Ploier R, Pendl G: Gamma knife radiosurgery for hypothalamic hamartomas in patients with medically intractable epilepsy and precocious puberty: report of two cases. J Neurosurg 92: 726-731, 2000

23) Valdueza JM, Cristante L, Dammann O, Bentele K, Vortmeyer A, Saeger W, Padberg B, Freitag J, Herrmann HD: Hypothalamic hamartomas: with special reference to gelastic epilepsy and surgery. Neurosurgery 34: 949-958, 1994

24) Villemure JG, Vernet O, Delalande O: Hemispheric disconnection: callosotomy and hemispherotomy. Adv Tech Stand Neurosurg 26: 25-78, 2000

Address reprint requests to: O. Delalande, M.D., Unité de neurochirurgie pédiatrique, Fondation Ophtalmologique A. de Rothschild, 25 rue Manin, 75019 Paris, France.

e-mail: odelalande@fo-rothschild.fr. 